Reprod. Nutr. Dévelop., 1988, 28 (2 A), 303-317.

\title{
Effets de la monensine sur la sécrétion des caséines dans les cellules épithéliales mammaires de lapines en lactation
}

\author{
Michèle OLLIVIER-BOUSQUET, Michèle AHMED-ALI
}

Laboratoire de Physiologie de la Lactation, I.N.R.A., 78350 Jouy-en-Josas, France.

Summary. Effects of monensin on milk casein secretion in lactating rabbit mammary gland fragments.

The effects in vitro of monensin on the secretory pathway of caseins in lactating rabbit mammary gland fragments were investigated. Addition of monensin $(0.1 \mu \mathrm{M}$ or $1 \mu \mathrm{M})$ to the incubation medium induced a dilatation of Golgi saccules and vesicles and a decrease of the relative volume of microvesicles. Dilated vesicles did not contain acid phosphatase. Neosynthesized proteins were localized by electron microscope autoradiography near the membranes of dilated vesicles. $1 \mu \mathrm{M}$ monensin did not inhibit basal secretion of neosynthesized caseins (radioactive caseins, labelled during a 3 min pulse, released into the medium), but increased basal secretion of total $\beta$-caseins (measured by radioimmunoassay). Monen$\sin$ abolished all the effects of prolactin (increase in the relative volume of Golgi microvesicles and stimulation of secretion of total and neosynthesized caseins).

These results show that monensin provokes simultaneously modifications in the Golgi apparatus morphology and inhibition of the prolactin stimulating effect. Moreover, secretion of intracellular caseins is differently modified, depending probably on whether caseins are neosynthesized or stored.

\section{Introduction.}

Le rôle de l'appareil de Golgi dans la synthèse et la sécrétion des caséines du lait a été souvent décrit (Bargmann et Knoop, 1958 ; Wellings et Pitelka, 1960 ; Wellings et Philp, 1964). Cet organite cellulaire est aussi impliqué lors du contrôle hormonal de la sécrétion : ainsi, l'ocytocine est capable d'accélérer le transit intracellulaire des caséines néosynthétisées de l'ergastoplasme vers l'appareil de Golgi et le mouvement des vésicules golgiennes vers l'apex de la cellule (OllivierBousquet et Denamur, 1976). La prolactine, qui exerce un effet rapide sur la sécrétion des caséines, provoque un accroissement du volume relatif des microvésicules de la région golgienne (Ollivier-Bousquet, 1978). Enfin, cette région constitue un lieu de circulation de membranes intracellulaires puisque des molécules endocytées après liaison à la membrane plasmique sont rapidement décelables dans la région golgienne (Kane et al., 1983). Cependant, les mécanismes précis du transport des protéines du lait et la régulation hormonale de ce transport dans 
cet organite sont peu clairs. L'ionophore carboxylique monovalent Ispécifique de $\mathrm{Na}^{+}$), la monensine, ralentit le transport intracellulaire des protéines et provoque une dilatation de l'appareil de Golgi dans de nombreuses cellules sécrétoires exocrines et endocrines (Tartakoff, 1983 ; Tougard et al., 1983). II a ainsi permis d'étudier la fonction golgienne et de nombreuses voies intracellulaires qui lui sont associées (Ledger et Tanzer, 1984 ; Melin et al., 1984 ; Burditt et al., 1985).

Dans ce travail, nous avons étudié l'effet de la monensine sur la morphologie des cellules épithéliales mammaires de lapines en lactation et sur la sécrétion des caséines stimulée ou non par la prolactine.

\section{Matériel et méthodes.}

Des fragments de glande mammaire de lapines néozélandaises (15e jour de lactation) sont prélevés immédiatement après abattage. Chaque expérience est réalisée avec des fragments de glande mammaire provenant d'une lapine.

Incubation. - Des fragments 10,1 à $0,2 \mathrm{mg}$ poids total pour chaque échantillon 10 à $30 \mathrm{mg}$ ) sont incubés dans un milieu de Hank's pH 7,$4 ; 37^{\circ} \mathrm{C}$; atmosphère $95 \% \mathrm{O}_{2}, 5 \% \mathrm{CO}_{2}$.

Marquage des caséines. - Les tissus sont préincubés pendant $30 \mathrm{~min}$ avec ou sans monensine (Sigma) (1 $\mu \mathrm{M}$ ), marqués pendant 3 min par $1480 \mathrm{kBq} / \mathrm{ml}$ de L-3-4-5 ${ }^{3} \mathrm{H}$-leucine (CEA, Saclay, $17760 \mathrm{GBq} / \mathrm{mmol}$ ), rincés abondamment dans le même milieu puis incubés pendant 60 min après le début du marquage en présence ou non de prolactine $(10 \mu \mathrm{g} / \mathrm{ml})$, de monensine $(1 \mu \mathrm{M})$ ou de l'association des deux. Il a été montré précédemment que ces conditions expérimentales de marquage et de rinçage sont celles qui permettent le mieux de mettre en évidence la stimulation prolactinique (Ollivier-Bousquet, 1978).

Dosage des protéines tissulaires marquées et des caséines radioactives sécrétées. - Les protéines tissulaires marquées et les caséines néosynthétisées radioactives sécrétées dans le milieu sont dosées selon la méthode décrite précédemment (Ollivier-Bousquet, 1978). Les tissus sont pesés, broyés dans $300 \mu \mathrm{l}$ de tampon phosphate salin $10 \mathrm{mM}$ pH 7,2 contenant $1 \%$ de triton et $0,5 \%$ de désoxycholate de sodium, précipités à l'aide de $300 \mu \mathrm{l}$ d'acide trichloroacétique $20 \%$ en présence de $50 \mu \mathrm{l}$ de sérum albumine $1 \%$. Les précipités sont lavés à l'acide trichloroacétique $10 \%$ et comptés en scintillation liquide dans un spectromètre Packard. La radioactivité obtenue est la radioactivité des protéines tissulaires $(\mathrm{cpm} / \mathrm{mg}$ de tissu). Les milieux d'incubation $(2 \mathrm{ml})$ sont filtrés sur millipores $3 \mu \mathrm{m}$ de façon à éliminer les débris cellulaires. Les filtres sont rincés par $1,5 \mathrm{ml}$ de Hank's. Les caséines sont précipitées à leur point isoélectrique par $1 \mathrm{ml}$ de tam-

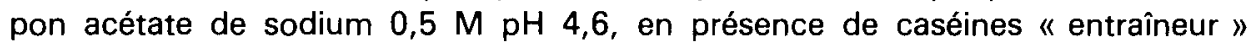
(dilution finale $0,5 \mathrm{mg} / \mathrm{ml}$ ). Les précipités sont lavés par le tampon acétate de sodium $0,5 \mathrm{M} \mathrm{pH} \mathrm{4,6} \mathrm{et} \mathrm{comptés} \mathrm{en} \mathrm{scintillation} \mathrm{liquide} \mathrm{dans} \mathrm{un} \mathrm{spectromètre}$ Packard. La radioactivité obtenue est la radioactivité des caséines sécrétées 
(cpm/mg de tissu). Les caséines radioactives sécrétées sont exprimées en pourcentage des protéines totales marquées selon la formule :

Caséines radioactives $\begin{aligned}(\%) & =\frac{\text { Radioactivité des caséines sécrétées }(\mathrm{cpm} / \mathrm{mg})}{\text { Radioactivité des caséines sécrétées }(\mathrm{cpm} / \mathrm{mg})} \times 100 . \\ & + \text { radioactivité des protéines tissulaires }(\mathrm{cpm} / \mathrm{mg})\end{aligned}$

Dosage des $\beta$-caséines totales sécrétées. - Les tissus sont préincubés pendant 15 min dans un milieu de Hank's afin d'effectuer un lavage puis pendant 30 min dans un nouveau milieu de Hank's avec ou sans monensine $(1 \mu \mathrm{M})$, enfin incubés pendant $60 \mathrm{~min}$ en présence ou non de prolactine $(10 \mu \mathrm{g} / \mathrm{ml})$, de monensine $(1 \mu \mathrm{M})$ ou de l'association des deux. Les $\beta$-caséines totales sécrétées dans le milieu sont dosées par radio-immuno-essai (Dusanter-Fourt et al., 1983).

Traitement des échantillons pour la microscopie électronique. - A la fin de l'incubation, les fragments sont fixés dans le paraformaldéhyde $4 \%$-glutaraldéhyde $4 \%$ (V/V) dans un tampon cacodylate $0,1 \mathrm{M}$, pendant $30 \mathrm{~min}$, puis dans le tétroxyde d'osmium $\left(\mathrm{OsO}_{4}\right) 1 \%$ dans le tampon cacodylate $0,1 \mathrm{M}$, pendant $2 \mathrm{~h}$, déshydratés et inclus dans l'Epon. Le volume relatif des différents constituants de l'appareil de Golgi est mesuré comme il a été décrit précédemment (Ollivier-Bousquet, 1978, 1980) : pour chaque expérience et pour chaque traitement, 20 micrographies $(\times 6300)$ contenant une ou deux cellules ont été analysées par la méthode des " comptages de points " grâce à une grille de points. Le volume relatif de chaque organite mesuré est le nombre de points comptés sur cet organite en pourcentage du nombre de points totaux comptés sur la cellule.

La localisation de la phosphatase acide est faite sur des échantillons fixés dans un mélange paraformaldéhyde $4 \%$ - glutaraldéhyde $1 \%(\mathrm{~V} / \mathrm{V})$ dans un tampon cacodylate $0,1 \mathrm{M}$ pendant 90 à $120 \mathrm{~min}$. Les échantillons sont ensuite incubés dans un milieu contenant du $\beta$-glycérophosphate de $\mathrm{Na}$ (Sigma, grade I) $3 \%$, du nitrate

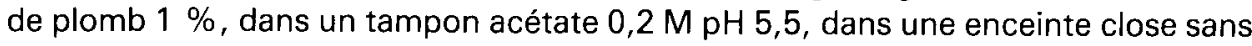
$\mathrm{CO}_{2}$ à $37^{\circ} \mathrm{C}$ pendant 60 à $90 \mathrm{~min}$ (Lewis, 1977). Après lavage, les échantillons sont post-fixés pendant $1 \mathrm{~h} 30$ dans $\mathrm{OsO}_{4} 1 \%$ dans un tampon cacodylate $0,1 \mathrm{M}$, déshydratés et inclus dans l'Epon.

Préparation des échantillons pour l'autoradiographie en microscopie électronique. Des fragments sont marqués pendant 3 min par $7400 \mathrm{kBq} / \mathrm{ml}$ de L-3 $\mathrm{H}$-leucine, lavés puis incubés comme précédemment en présence ou non de prolactine $(10 \mu \mathrm{g} / \mathrm{ml})$ et de monensine $(1 \mu \mathrm{M})$ ou de l'association des deux ; 60 min après le début du marquage, les échantillons sont fixés et traités comme il a été décrit précédemment pour la localisation de la phosphatase acide, post-fixés par $\mathrm{OsO}_{4} 1 \%$ dans le tampon cacodylate $0,1 \mathrm{M}$, déshydratés et inclus dans I'Epon. Les coupes sont recouvertes avec un film de carbone et une couche d'émulsion llford L4, exposées pendant 21 jours et développées avec du microdol $X$.

Analyse statistique. - Chaque expérience est faite à partir de fragments de glande mammaire provenant d'une lapine. Dans une expérience, le témoin et le traité ne diffèrent que par l'addition de l'hormone ou de l'ionophore. La comparai-

Reproduction, Nutrition, Développement $n^{\circ} 2 \mathrm{~A}-88 .-7$ 


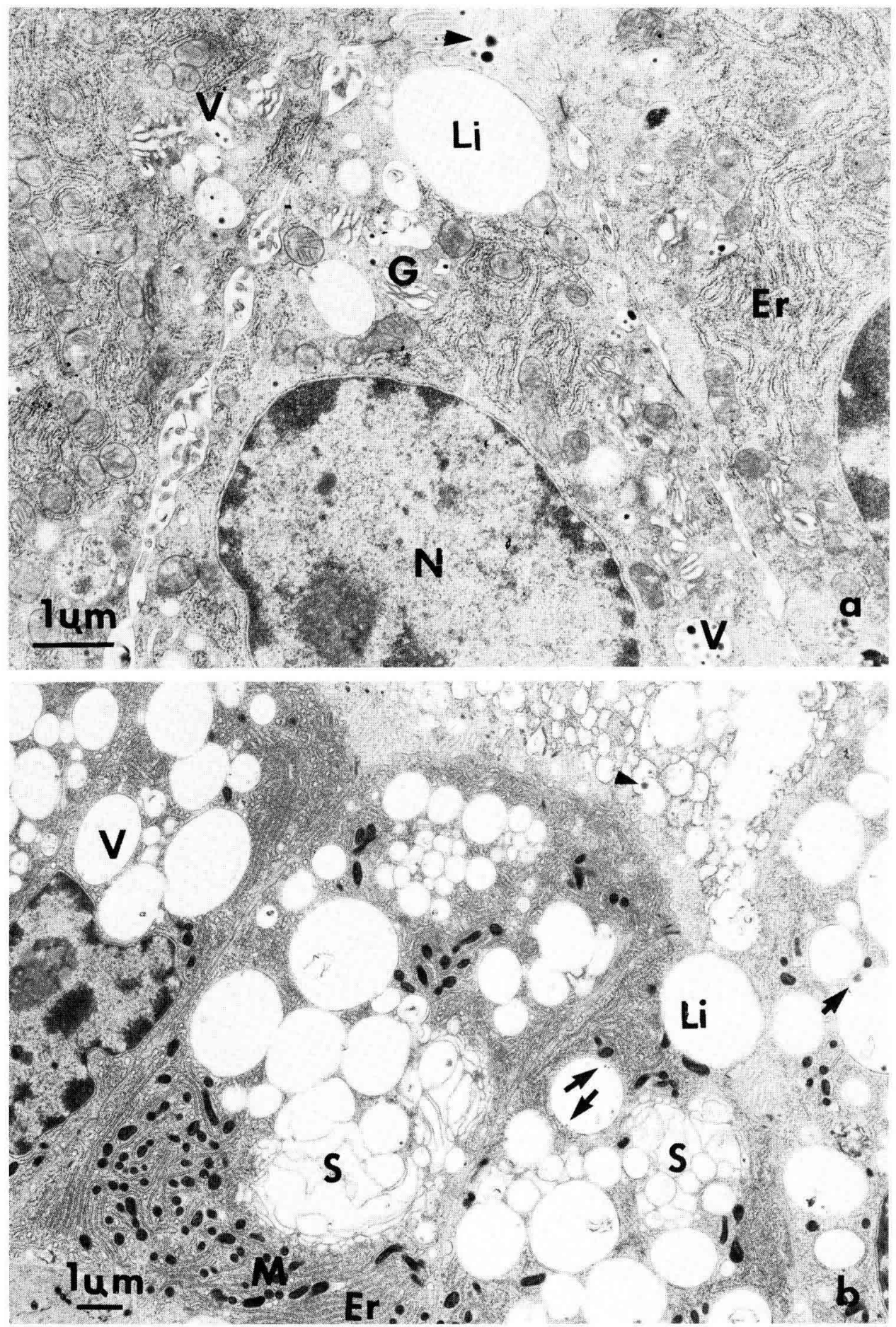


son entre les couples de valeur provenant du même animal a été faite par un test $\mathrm{t}$ de Student sur les différences appariées.

\section{Résultats.}

Effets de la monensine sur les cellules épithéliales mammaires.

Effets morphologiques. - Après une heure d'incubation en présence de monensine $(0,1 \mu \mathrm{M}$ et $1 \mu \mathrm{M})$, l'organisation générale du tissu mammaire en acini n'est pas modifiée. Les lumières des acini contiennent des produits de sécrétion (protéines et lipides) ainsi que de nombreux constituants cellulaires. Cependant, toutes les cellules épithéliales mammaires présentent un aspect vacuolaire (fig. 1). Les saccules golgiens sont très dilatés et de nombreuses vésicules entourant la région golgienne envahissent tout le cytopıasme. Ces vésicules, vides pour la plupart, sont entourées d'une membrane présentant des portions plus ou moins épaissies. Cette membrane permet de faire la différence entre les vésicules dilatées et les globules lipidiques. Ces derniers ne présentent pas de membrane tant qu'ils sont intracellulaires. Certaines vésicules contiennent un ou deux micelles de caséines (Bargmann et Knoop, 1958; Burditt et al., 1985) ou un matériel filamenteux adhérant à la membrane. Les mitochondries, très nombreuses, sont souvent très denses. Enfin l'ergastoplasme en lamelles parallèles n'est pas modifié par la monensine.

En présence de monensine $(1 \mu \mathrm{M})+$ prolactine $(10 \mu \mathrm{g} / \mathrm{ml})$, l'aspect des cellules est identique à celui décrit en présence de monensine seule.

Mesure du volume relatif des constituants de l'appareil de Golgi. - Le volume occupé par les microvésicules (diamètre des vésicules de 50 à $300 \mathrm{~nm}$ environ) (fig. 2 et 3), fortement augmenté en présence de prolactine, est réduit en présence de monensine. Dans ce cas, le cytoplasme situé à la périphérie de I'appareil de Golgi contient quelques microvésicules dispersées dans un cytosol dense et filamenteux. Le volume relatif des vésicules entourées par une membrane pouvant être assimilées aux vésicules de sécrétion est fortement augmenté en présence de monensine $0,1 \mu \mathrm{M}$ et $1 \mu \mathrm{M}$ et de l'association monensine + pro-

FIG. 1. - Cellules épithéliales mammaires de lapines en lactation.

a : Cellules épithéliales incubées pendant $60 \mathrm{~min}$ dans un milieu de Hank's. La lumière contient des caséines ( ). Un globule lipidique (Li), à l'apex de la cellule est en cours de sécrétion.

Ergastoplasme $(\mathrm{Er})$; noyau $(\mathrm{N})$; appareil de Golgi $(\mathrm{G})$; vésicules de sécrétion contenant des caséines (V). × 11300 .

b : Cellules épitheliales incubées pendant 60 min dans un milieu de Hank's + monensine $(1 \mu \mathrm{M})$. La lumière contient quelques caséines $(\boldsymbol{D})$ et de nombreux débris cellulaires. Un globule lipidique (Li) est en cours de sécrétion. Les saccules de l'appareil de Golgi (S) sont très dilatés. De nombreuses vésicules (V) remplissent le cytoplasme. La plupart apparaissent vides. Certaines contiennent des caséines $(\rightarrow$ ) ou un matériel filamenteux adhérent à la membrane. L'ergastoplasme (Er) est très développé. Les mitochondries $(\mathrm{M})$ sont très denses. $\times 6300$. 


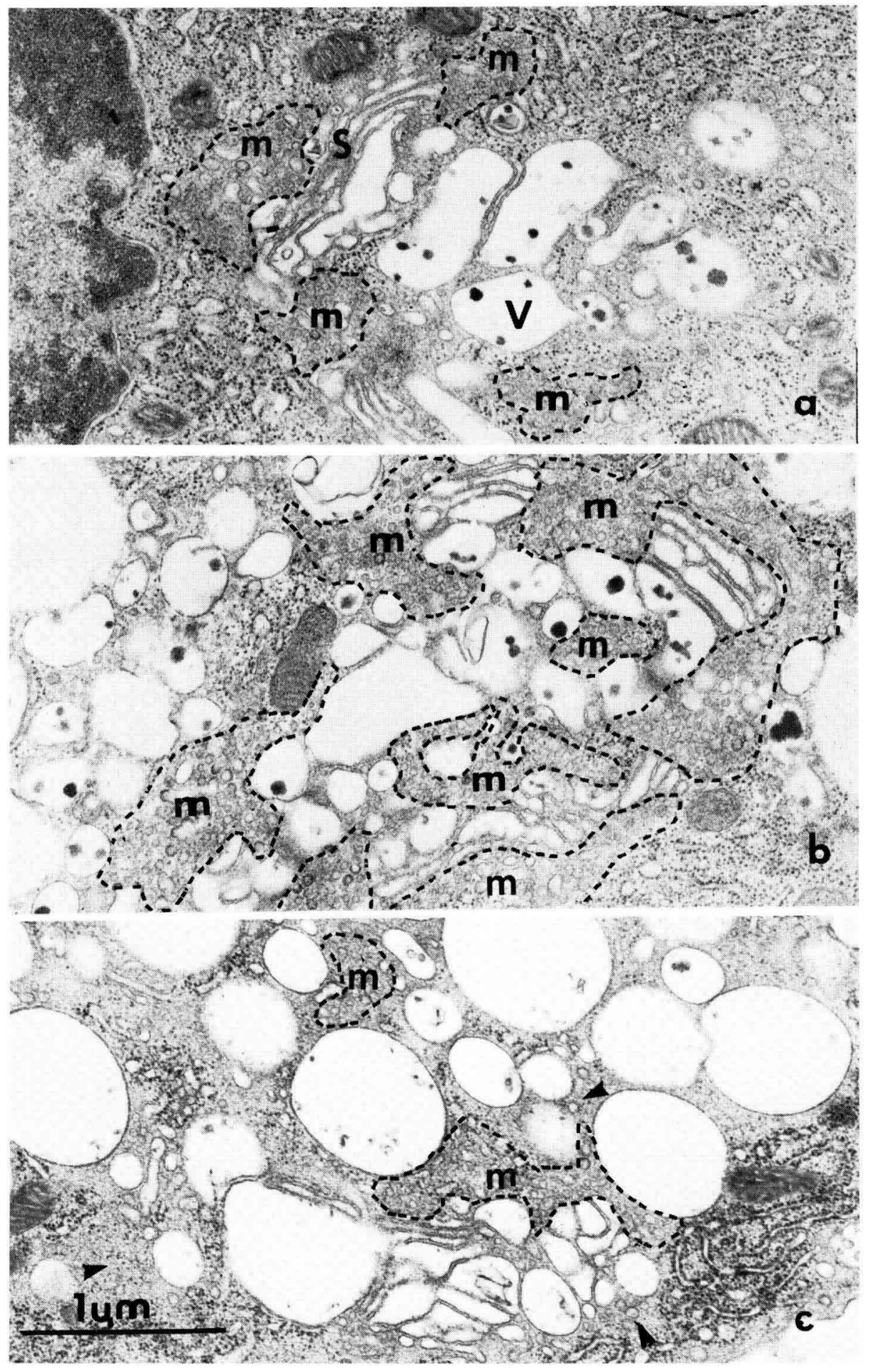


lactine. De même, le volume relatif des saccules est augmenté sous l'effet de la monensine (fig. 3).

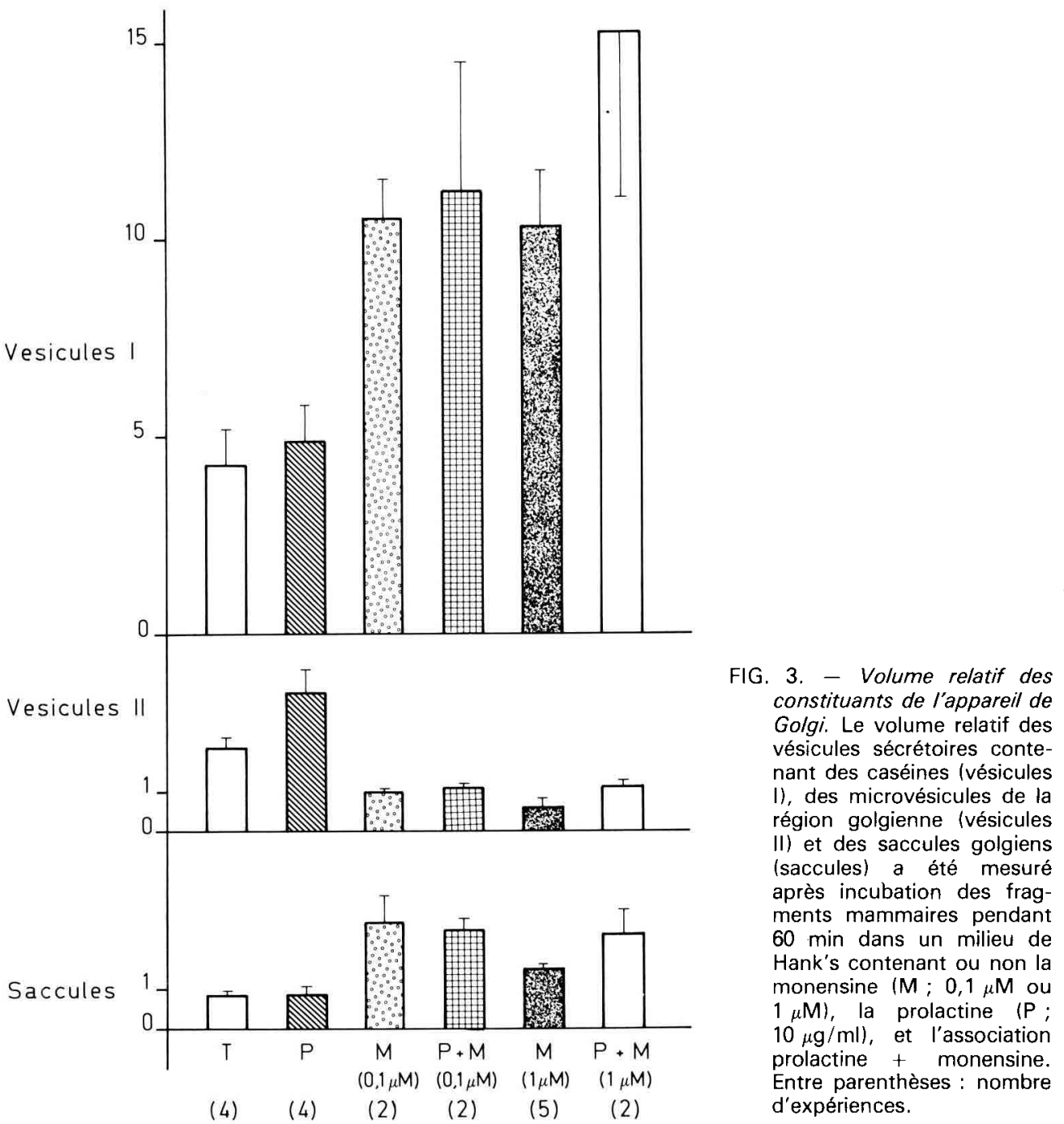

FIG. 2. - Région golgienne de cellules épithéliales mammaires de lapines en lactanu." La surface cytoplasmique occupée par les microvésicules est entourée (---).

a : Cellule epithéliale de fragment mammaire incubé pendant 60 min dans un milieu de Hank's. Saccules (S), microvésicules $(\mathrm{m})$ et vésicules (V) contenant des caséines. $\times 29300$.

b : Cellule épithéliale de fragment mammaire incubé pendant $60 \mathrm{~min}$ dans un milieu de Hank's + prolactine $(10 \mu \mathrm{g} / \mathrm{ml})$. La surface occupée par les microvésicules $(\mathrm{m})$ est fortement augmentée. $\times 29000$.

c : Cellule épithéliale de fragment mammaire incubé pendant 60 min dans un milieu de Hank's + monensine $(1 \mu \mathrm{M})$. La surface occupée par les microvésicules $(\mathrm{m})$ est réduite. Des plages de cytoplasme dense et filamenteux $(\rightarrow$ apparaissent entre les vésicules (V) dilatées. Quelques microvésicules sont éparses dans la région golgienne ( $) \times 29000$. 
Localisation des phosphatases acides. - Afin de préciser la nature des vésicules dilatées sous l'effet de la monensine, les phosphatases acides ont été localisées dans les fragments mammaires après les différents traitements par la monensine et la prolactine (fig. 4). Dans les tissus témoins, quelques vésicules contenant des fragments membranaires, situés à proximité de l'appareil de Golgi, sont réactives. La prolactine provoque une augmentation du nombre de ces vésicules. En présence de monensine, des vésicules réactives du même type sont décelables. Cependant, les vésicules dilatées ne présentent pas de réaction.

Localisation par autoradiographie des protéines néosynthétisées. - La localisation simultanée des protéines intracellulaires néosynthétisées marquées par

\section{TABLEAU 1}

Effet de la monensine $(1 \mu \mathrm{M})$ sur la synthèse protéique totale. Les fragments de glande mammaire ont été incubés pendant 30 min dans un milieu de "Hank's " contenant ou non de la monensine $1 \mu \mathrm{M}$, marqués pendant $3 \mathrm{~min}$ dans un milieu contenant $1480 \mathrm{kBq} / \mathrm{ml}$ de L ${ }^{3} \mathrm{H}$-leucine, lavés abondamment, puis incubés pendant $60 \mathrm{~min}$ dans le milieu de Hank's contenant de la prolactine $10 \mu \mathrm{g} / \mathrm{ml}$, de la monensine $(1 \mu \mathrm{M})$ ou l'association des deux. La synthèse protéique totale est mesurée après précipitation des tissus au TCA $10 \%$. Moyennes \pm ES - 5 expériences.

\begin{tabular}{|c|c|c|}
\hline Milieu de préincubation & Milieu d'incubation & $\begin{array}{l}\text { Radioactivité des tissus } \\
(\mathrm{cpm} / \mathrm{mg})\end{array}$ \\
\hline Hank's & Hank's & $23798 \pm 5331$ \\
\hline Hank's & $\begin{array}{l}\text { Hank's }+ \text { prolactine } \\
10 \mu \mathrm{g} / \mathrm{ml}\end{array}$ & $20424 \pm 4556$ \\
\hline Hank's & Hank's + monensine $1 \mu \mathrm{M}$ & $24224 \pm 5270$ \\
\hline Hank's & $\begin{array}{l}\text { Hank's + monensine } 1 \mu \mathrm{M} \\
+ \text { prolactine } 10 \mu \mathrm{g} / \mathrm{ml}\end{array}$ & $21675 \pm 3235$ \\
\hline Hank's + monensine $1 \mu \mathrm{M}$ & Hank's + monensine $1 \mu \mathrm{M}$ & $23113 \pm 5451$ \\
\hline Hank's + monensine $1 \mu \mathrm{M}$ & $\begin{array}{l}\text { Hank's }+ \text { monensine } 1 \mu \mathrm{M} \\
\quad+\text { prolactine } 10 \mu \mathrm{g} / \mathrm{ml}\end{array}$ & $21105 \pm 2982$ \\
\hline
\end{tabular}

FIG. 4. - Mise en évidence des phosphatases acides dans les cellules épithéliales mammaires de lapines en lactation (pas de coloration au citrate de plomb).

a : Région golgienne d'une cellule épithéliale incubée pendant 60 min dans un milieu de Hank's. Le produit de réaction est localisé dans une vésicule contenant des débris membranaires $(\rightarrow$. Les saccules golgiens $(S)$ et les vésicules contenant des caséines ne sont pas réactifs. Une vésicule contient à la fois des fragments membranaires réactifs et des caséines $(\vec{\rightarrow})$. Les lipides présentent une réaction périphérique non spécifique. $\times 30000$.

$\mathrm{b}$ : Région golgienne d'une cellule épithéliale incubee pendant 60 min dans un milieu de Hank's + prolactine $(10 \mu \mathrm{g} / \mathrm{ml})$. Les produits de réaction sont décelables dans de nombreuses vésicules contenant des débris cellulaires et des fragments membranaires $(\rightarrow$. Les saccules golgiens (S) et les vésicules contenant les caséines $(V)$ ne sont pas réactifs. $\times 20000$.

$c$ : Région golgienne d'une cellule épithéliale incubée pendant 60 min dans un milieu de Hank's + monensine $(1 \mu \mathrm{M})$. Les saccules (S) et les vésicules (V) ne sont pas réactifs. Cependant un petit nombre de vésicules $(\rightarrow$ ) contiennent à la fois des fragments membranaires présentant une réaction et des caséines, comme dans les témoins. $\times 20000$. 

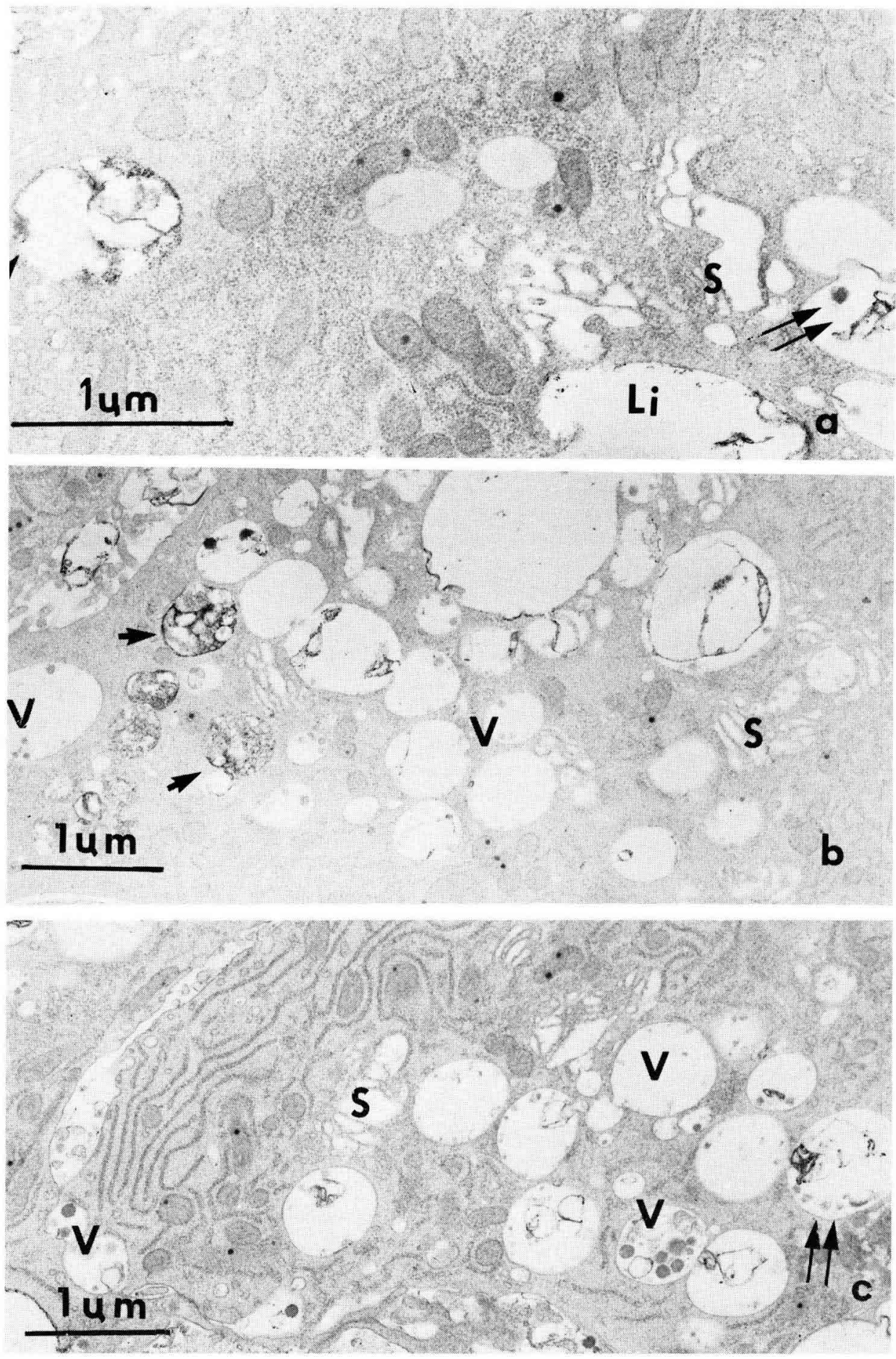
autoradiographie en microscopie électronique et des phosphatases acides (fig. 5), montre que, après incubation en présence de monensine, de nombreux grains d'argent sont localisés à la périphérie des vésicules dilatées qui ne réagissent pas à la réaction de mise en évidence des phosphatases acides. Les vésicules sont donc assimilables aux vésicules golgiennes contenant des caséines en cours de synthèse.
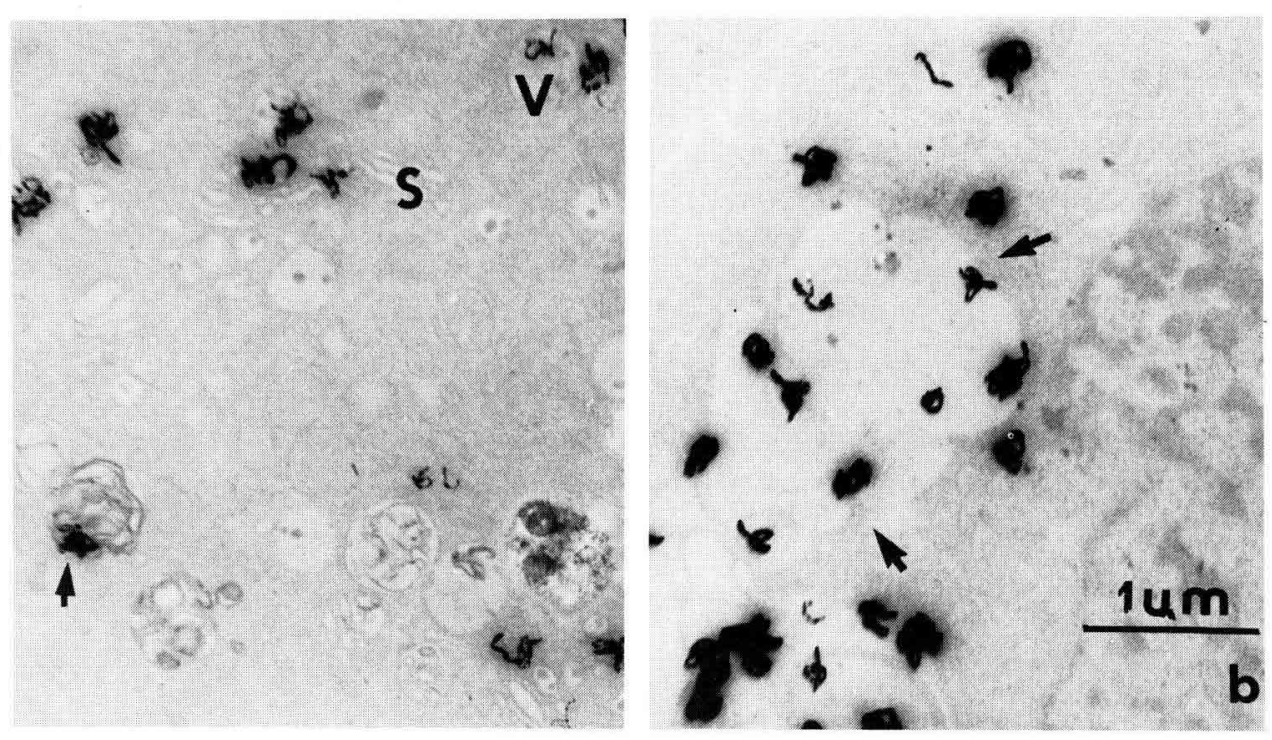

FIG. 5. - Localisation autoradiographique des protéines néosynthétisées dans des fragments mammaires marqués pendant $3 \mathrm{~min}$ par la L-3H-leucine, lavés abondamment, incubés dans un milieu de Hank's pendant 60 min, fixés et traités pour la localisation des phosphatases acides puis postfixés et inclus (pas de coloration au citrate de plomb).

a : Cellules épithéliales incubées dans un milieu de Hank's + prolactine $(10 \mu \mathrm{g} / \mathrm{ml})$. Des grains d'argent sont situés sur les éléments de I'appareil de Golgi (vésicules : $V$; saccules : S). Une vésicule contenant des produits de réaction aux phosphatases acides est marquée $(\rightarrow)$. $\times 12600$. b : Cellules épithéliales incubées dans un milieu de Hank's + monensine $(1 \mu \mathrm{M})$. Les vésicules dilatées ne contenant pas de produit de réaction aux phosphatases acides, sont marquées à leur périphérie $(\rightarrow) \times 12600$.

Effets de la monensine sur la sécrétion des caséines.

Synthèses protéiques. - Le tableau 1 montre les effets de la monensine sur les synthèses protéiques dans les fragments de glande mammaire en incubation. La monensine $1 \mu \mathrm{M}$ ajoutée soit dans le milieu d'incubation, soit dans le milieu de préincubation puis d'incubation, ne provoque pas de modification de la radioactivité des tissus.

Sécrétion des caséines néosynthétisées. - La monensine $(1 \mu \mathrm{M})$, soit rajoutée après le marquage, soit présente dans la préincubation puis après le mar- 
quage, ne modifie pas la sécrétion de base mais inhibe la stimulation prolactinique de la sécrétion des caséines néosynthétisées, marquées au cours d'un " pulse ", après 60 min d'incubation (fig. 6).

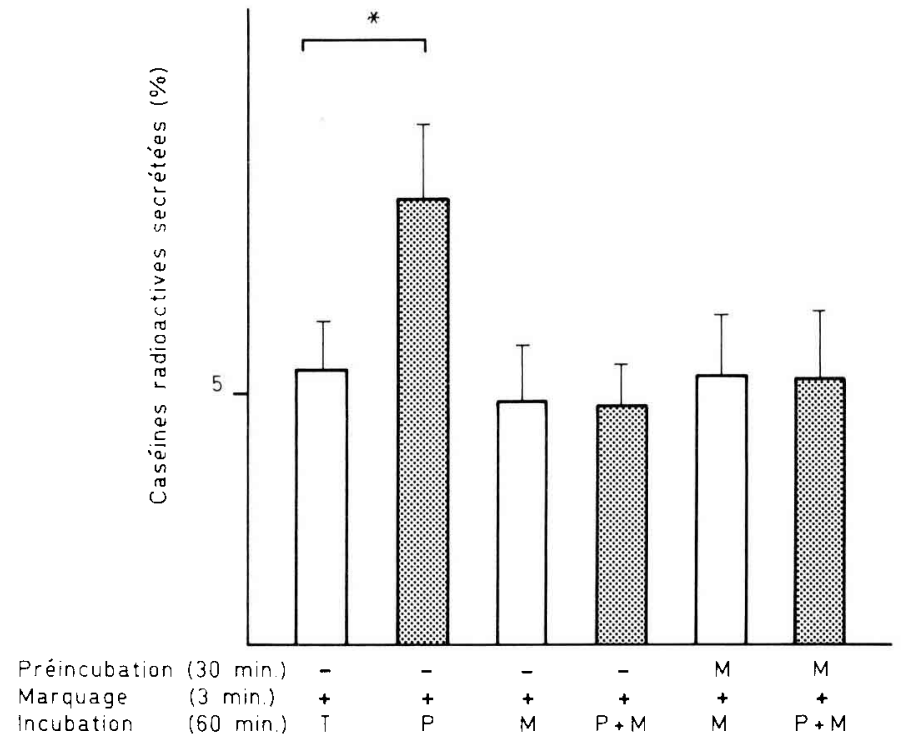

FIG. 6. - Effet de la monensine $(1 \mu \mathrm{m})$ sur la sécrétion de base et la sécrétion stimulée par la prolactine, des caséines néosynthétisées. Les fragments de tissu mammaire sont préincubés pendant 30 min, dans un milieu de Hank's $(T)$ contenant ou non de la monensine $(\mathrm{M} ; 1 \mu \mathrm{M})$, marqués pendant 3 min par la L- ${ }^{3} \mathrm{H}$-leucine, lavés abondamment puis incubés avec ou sans monensine en présence ou non de prolactine $(P)(10 \mu \mathrm{g} / \mathrm{ml}) .60 \mathrm{~min}$ après le début du marquage, les caséines radioactives sécrétées dans le milieu sont mesurées comme décrit dans matériel et méthodes. Moyennes \pm ES -18 expériences. ${ }^{* *} p<0,02$.

Afin de voir à quel niveau du transit intracellulaire la monensine inhibe la stimulation prolactinique, l'ionophore a été rajouté à différents temps après le pulse (tabl. 2). II apparaît clairement que la monensine n'a plus aucun effet inhibiteur sur la stimulation prolactinique dès lors qu'elle est rajoutée $10 \mathrm{~min}$ après le début du marquage, la prolactine étant elle-même rajoutée dès la fin du marquage et du rinçage, c'est-à-dire entre 3 et 5 min après le début du marquage.

Sécrétion des $\beta$-caséines totales. - Lorsque la monensine est rajoutée pendant $60 \mathrm{~min}$ dans le milieu d'incubation, la quantité totale de $\beta$-caséines sécrétées dans le milieu, mesurée par RIA, est augmentée. L'association monensine $(1 \mu \mathrm{M})$ + prolactine $(10 \mu \mathrm{g} / \mathrm{ml})$ provoque la même augmentation que la monensine seule.

Quand les échantillons sont préincubés pendant $30 \mathrm{~min}$ en présence de monensine $(1 \mu \mathrm{M})$ puis incubés dans un nouveau milieu contenant de la monen- 
sine $(1 \mu \mathrm{M})$, le niveau de base de la sécrétion des $\beta$-caséines n'est pas modifié mais la stimulation prolactinique de la sécrétion est complètement inhibée (fig. 7).

\section{TABLEAU 2}

Effet de la monensine $(1 \mu \mathrm{M})$ rajoutée à différents temps après le marquage. Les fragments de glande mammaire ont été marqués pendant $3 \mathrm{~min}$ dans un milieu contenant $1480 \mathrm{kBq} / \mathrm{ml}$ de L ${ }^{3} \mathrm{H}$ leucine, lavés abondamment, puis incubés pendant 60 ou $90 \mathrm{~min}$ dans le milieu de Hank's contenant de la prolactine $(10 \mu \mathrm{g} / \mathrm{ml})$, de la monensine $(1 \mu \mathrm{M})$ ou l'association des deux, soit à partir de la fin du marquage, soit à des temps différents après le marquage. L'introduction respective de la prolactine et de la monensine est indiquée par une flèche / Prol; $(\mathrm{M})$ ). Les caséines radioactives sécrétées dans le milieu sont mesurées comme décrit dans matériels et méthodes. Moyennes $\pm E S-4$ expériences. ${ }^{*} p<0,05 ;{ }^{*} p<0,02$.

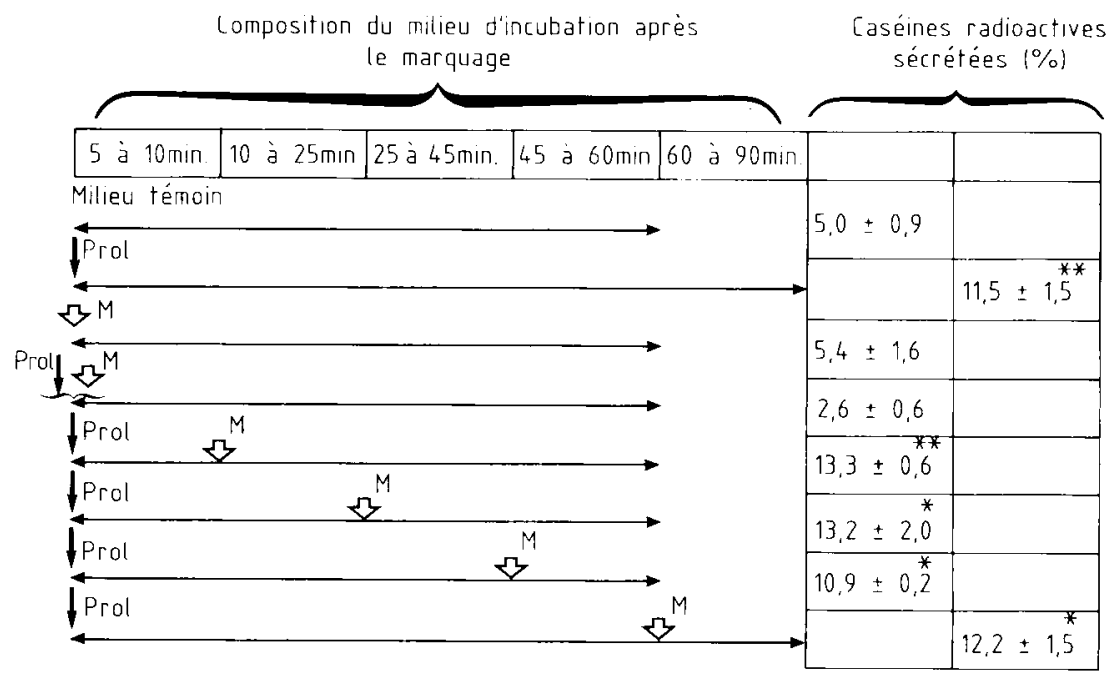

\section{Discussion.}

Les effets de la monensine sur la morphologie des cellules épithéliales mammaires sont analogues à ce qui a été décrit dans de nombreuses cellules sécrétoires (Tartakoff et Vassili, 1978 ; Tartakoff, 1983 ; Tougard et al., 1983 : Orci et al., 1984). Dans les cellules épithéliales mammaires, les vésicules dilatées ne contiennent pas de phosphatase acide mais des protéines néosynthétisées, et peuvent donc être assimilées à des vésicules golgiennes contenant des produits de sécrétion. Ceci est en accord avec les effets de la monensine dans les cellules $\mathrm{GH} 3$ (Tougard et al., 1983) où la prolactine en cours de sécrétion reste attachée à la face interne de la membrane des vésicules dilatées. Dans les cellules pancréatiques, il a été montré, par autoradiographie en microscopie électronique, que la monensine agit au point de sortie des protéines sécrétoires, hors de l'appareil de Golgi (Tartakoff et Vassili, 1978). La monensine est responsable du blocage de la proinsuline dans un compartiment de membranes épaissies par la clathrine alors que le transit normal s'effectue par une maturation postérieure dans un comparti- 


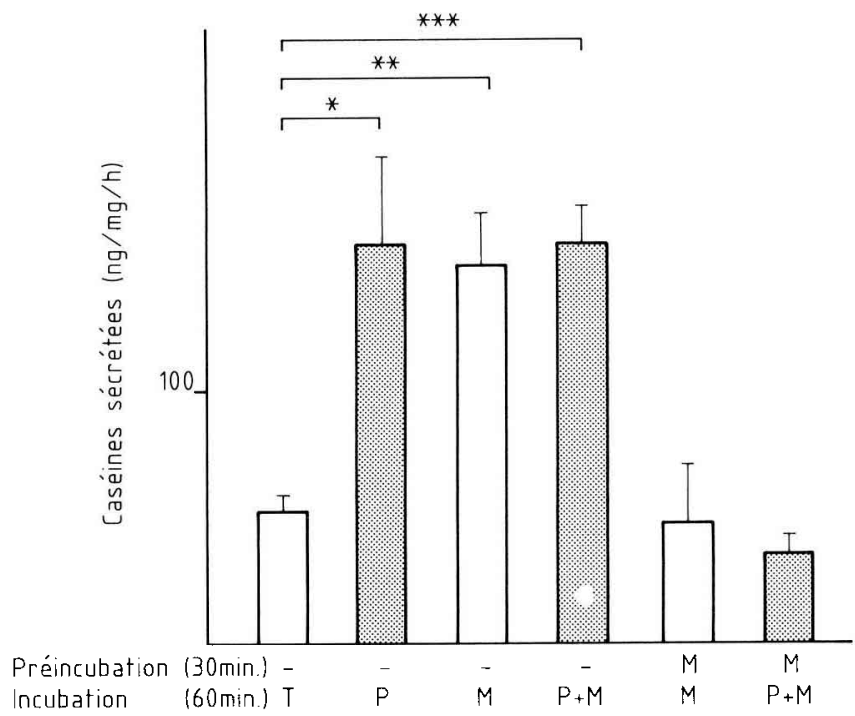

FIG. 7. - Effet de la monensine $(1 \mu \mathrm{M})$ sur la sécrétion de base et la sécrétion stimulée par la prolactine, des caséines totales. Les fragments de tissu mammaire sont préincubés pendant 30 min dans un milieu de Hank's (T) contenant ou non de la monensine $(\mathrm{M} ; 1 \mu \mathrm{M})$ puis incubés dans un milieu de Hank's, avec ou sans monensine, en présence ou non de prolactine ( $\mathbf{P} ; 10 \mu \mathrm{g} / \mathrm{ml}$ ). Après $60 \mathrm{~min}$ d'incubation, les caséines totales sécrétées sont mesurées dans les milieux comme décrit dans matériel et méthodes. Moyennes \pm ES -9 expériences. ${ }^{*} p<0,02$; ${ }^{*} p<0,01$; ${ }^{* * *} \mathrm{p}<0,001$.

ment membranaire sans clathrine suivi par la libération de l'insuline (Orci et al., 1984). Dans les cellules épithéliales mammaires, les différents constituants de l'appareil de Golgi (vésicules, saccules, microvésicules) sont modifiés par la monensine. Les caséines marquées néosynthétisées sont décelables dans ces compartiments golgiens et, en particulier, à la périphérie des vésicules golgiennes dilatées. Parallèlement, la stimulation prolactinique de la sécrétion est inhibée. Ceci suggère que la stimulation prolactinique de la sécrétion des caséines pourrait s'exercer au cours d'une étape golgienne.

Parallèlement aux modifications morphologiques, la monensine interagit avec la sécrétion des caséines. La sécrétion de base des caséines néosynthétisées n'est pas modifiée alors que la sécrétion de base des caséines totales est fortement augmentée. On peut penser que ces caséines totales représentent en grande partie les caséines synthétisées et stockées avant le début du traitement par la monensine. Dans le cas où les tissus sont préincubés en présence de monensine, ces caséines sont sécrétées dans le premier milieu de préincubation et les caséines totales mesurées dans le nouveau milieu ne représentent plus que les caséines néosynthétisées. On peut faire I'hypothèse que la monensine, en favorisant par son effet ionophore l'entrée de $\mathrm{Na}^{+}$(Pressmann, 1968), pourrait stimuler la sécrétion des caséines totales stockées dans le tissu. Cependant, le rôle de $\mathrm{Na}^{+}$dans la sécrétion des caséines n'est pas bien connu. II a été décrit que la prolactine active l'extrusion de $\mathrm{Na}^{+}$dans les cellules mammaires (Falconer et Rowe, 1977). 
Le passage de ${ }^{24} \mathrm{Na}$ dans le lait varie selon l'état physiologique (Linzell et Peaker, 1974). Des travaux supplémentaires sont nécessaires pour élucider le rôle de $\mathrm{Na}^{+}$ dans la cellule mammaire.

Enfin, aussi bien pour les caséines néosynthétisées que pour les caséines totales, la monensine inhibe l'action stimulante de la prolactine.

Les effets de la monensine sur la sécrétion des caséines néosynthétisées sont analogues aux effets observés sur la sécrétion d'insuline stimulée par le glucose : dans les îlots de Langerhans isolés, la monensine ne semble pas avoir d'effet sur la libération de base de l'insuline mais inhibe la sécrétion stimulée par $20 \mathrm{mM}$ de glucose (Smith et Howell, 1984). Dans d'autres tissus, les effets de la monensine sont variables. Ainsi, dans la lignée cellulaire PC 12, synthétisant et sécrétant la dopamine $\beta$-hydroxylase, la monensine bloque le transport de protéines de haut poids moléculaire dans l'appareil de Golgi mais n'inhibe pas la stimulation par des sécrétagogues de la libération d'un groupe de protéines de bas poids moléculaire déjà prêtes à être sécrétées (Khun et al., 1986). Enfin, la monensine inhibe le niveau de base de la sécrétion de prolactine par les cellules $\mathrm{GH} 3$, mais n'inhibe pas la libération de prolactine induite par le TRH (Tougard et al., 1983). Dans ce cas, les cellules contiennent très peu de granules sécrétoires et il a été suggéré que la libération stimulée de la prolactine en réponse au TRH concerne la prolactine contenue dans de petites vésicules. Dans ces cellules pituitaires normales ou tumorales, la prolactine néosynthétisée est libérée rapidement dans les conditions de base avec deux " pools " de demi-vie différents. La TRH induit la libération préférentielle d'un " pool » de prolactine synthétisé avant le marquage (Morin et al., 1984a,b).

Dans la cellule mammaire, les résultats obtenus montrent que la monensine inhibe la sécrétion stimulée des caséines mais que cette sécrétion stimulée est insensible à la monensine si on fait agir cette dernière après que les caséines marquées aient déjà parcouru un trajet intracellulaire et que la prolactine ait eu le temps d'exercer un effet. On peut donc émettre l'hypothèse que la sécrétion des caséines ayant subi tous les processus post-traductionnels et situés dans un compartiment de stockage post-golgien ne serait pas inhibée par la monensine. Au contraire, la stimulation de la sécrétion des caséines en cours de transit serait sensible à la monensine. L'effet de l'ionophore sur la morphologie de l'appareil de Golgi, parallèlement à l'inhibition de la stimulation prolactinique, s'avère intéressant pour l'étude de la stimulation hormonale de la sécrétion exocrine.

Reçu en septembre 1987.

Accepté en novembre 1987.

Remerciements. - Nous remercions très vivement Mme le Docteur A. Tixier Vidal et $M$. le Professeur H. Clauser pour leurs conseils et leurs critiques ainsi que M. Grabowski pour le dosage des caséines par radio-immunoessai et Mme J. Brugnolo pour la préparation du manuscrit. 


\section{Róférences}

BARGMANN W., KNOOP A., 1958. Uber die Morphologie der Milchsekretion, Licht- und elektronenmikroskopische Studien an der Milchdrüse der Ratte. Z. Ze/lforsch, 49, 344-388.

BURDITT L. J., RATCLIFFE A., FRYER P. R., HARDINGHAM T. E., 1985. The intracellular localisation of proteoglycans and their accumulation in chondrocytes treated with monensin. Biochim. Biophys. Acta, 844, 247-255.

DUSANTER-FOURT I., DJIANE J., HOUDEBINE L. M., KELLY P., 1983. In vivo lactogenic effects of anti-prolactin receptor antibodies in pseudopregnant rabbits. Life Sci., 32, 407-412.

FALCONER I. R., ROWE J. M., 1977. Effect of prolactin on sodium and potassium concentrations in mammary alveolar tissue. Endocrinology, 101, 181-186.

KANE S., RAYMOND M. N., DUSANTER-FOURT I., HOUDEBINE L. M., DJIANE J., OLLIVIERBOUSQUET M., 1983. Endocytose de la prolactine dans la cellule épithéliale mammaire : effets des agents lysosomotropes et des inhibiteurs de la transglutaminase. Eur. J. Cell. Biol., 30, 244-253.

KUHN L. J., HADMAN M., SABBAN E. L., 1986. Effect of monensin on synthesis, posttranslational processing and secretion of dopamine $\beta$-hydroxylase from PC 12 pheochromocytoma cells. J. biol. Chem., 261, 3816-3825.

LEDGER P. W., TANZER M. L., 1984. Monensin a perturbant of cellular physiology. Trends biol. Sci., 103, 313-314.

LEWIS P. R., 1977. Metal precipitation methods for hydrolytic enzymes. In LEWIS P. R., KNIGHT D. P. Staining methods for sectionned material, 137-223, North-Holland, Amsterdam.

LINZELL J. L., PEAKER M., 1974. Changes in colostrum composition and in the permeability of the mammary epithelium at about the time of parturition in the goat. J. Physiol., 243, 129-151.

MELIN B., KELLER G., GLASS C., WEINSTEIN D. B., STEINBERG D., 1984. Lipoprotein synthesis and secretion by cultured rat hepatocytes. Biochim. Biophys. Acta, 795, 574-588.

MORIN A., ROSENBAUM E., TIXIER-VIDAL A., 1984a. Effects of thyrotropin - releasing hormone on prolactin compartments in clonal rat pituitary tumor cells. Endocrinology, 115, 22;22277.

MORIN A., ROSENBAUM E., TIXIER-VIDAL A., 1984b. Effects of thyrotropin - releasing hormone on prolactin compartments in normal rat pituitary cells in primary cultures. Endocrinology, 115, 2278-2284.

OLLIVIER-BOUSQUET M., 1978. Early effects of prolactin on lactating rabbit mammary gland. Ultrastructural changes and stimulation of casein secretion. Cell. Tiss. Res., 187, 25-43.

OLLIVIER-BOUSQUET M., 1980. Effet des agents lysosomotropes sur la stimulation par la prolactine de la sécrétion des protéines du lait. Biol. cell., 39, 21-30.

OLLIVIER-BOUSQUET M., DENAMUR R., 1976. Effet de l'ocytocine in vitro sur le transit intracellulaire et la sécrétion des protéines du lait. C. R. Acad. Sci., Paris, 282, 1433-1434.

ORCI L., HALBAN P., AMHERDT M., RAVAZZOLA M., VASSALI J. D., PERRELET A., 1984. A coated golgi related compartment of the insulin secreting cell accumulates proinsulin in the presence of monensin. Cell., 39, 39-47.

PRESSMANN B. C., 1968. Ionophorous antibiotics as models for biological transport. Fed. Proc., 27, $1283-1288$.

SMITH I. E., HOWELL S. L., 1984. Effects of monensin on metabolism of isolated rat islets of langerhaus. Biochem. J., 223, 423-432.

TARTAKOFF A. M., 1983. Perturbation of vesicular traffic with the carboxylic ionophore monensin. Cell., 32, 1026-1028.

TARTAKOFF A. M., VASSILI P., 1978. Comparative studies of intracellular transport of secretory proteins. J. Cell Biol., 79, 694-707.

TOUGARD C., PICART R., MORIN A., TIXIER-VIDAL A., 1983. Effect of monensin on secretory pathway in GH3 prolactin cells. J. Histochem. Cytochem., 31, 745-754.

WELLINGS S. R., PITELKA D. R., 1960. Electron microscopy of milk secretion in the mammary gland of $\mathrm{CrH} / \mathrm{Crgl}$ mouse. I. Cytomorphology of the prelactating and lactating gland. J. nat. Cancer Inst., 25, 393-421.

WELLINGS S. R., PHILP J. R., 1964. The function of the Golgi apparatus in lactating cells of the Balb/Crgl mouse. An electron microscopic and autoradiographic study. Z. Zellforsch, 61, 871-882. 